\title{
Costs of smoking during pregnancy: development of the Maternal and Child Health Smoking Attributable Mortality, Morbidity and Economic Costs (MCHSAMMEC) software
}

\author{
Cathy L Melvin, E Kathleen Adams, Vince Miller
}

Cecil G Sheps Center for Health Services Research, University of North Carolina at Chapel Hill, North Carolina, USA C L Melvin

Rollins School of Public Health, Emory University, USA E K Adams

Berkeley Economic Research Associates, Berkeley, California, USA

V Miller

Correspondence to: Cathy L Melvin, PhD, MPH Cecil G Sheps Center for Health Services Research, CB 7590, 725 Airport Road, University of North Carolina, Chapel Hill, NC 27599-7590, USA; cathy_melvin@unc.edu

Received 6 December 1999 and in revised form 2 March 2000

Accepted 16 March 2000

\begin{abstract}
The development and availability is described of new, user friendly software, the Maternal and Child Health Smoking Attributable Mortality, Morbidity and Economic Costs (MCHSAMMEC), that will allow states and other entities to estimate pregnancy related, smoking attributable costs for their population. The methodology underlying the MCHSAMMEC software, including calculations used in the prevalence based analysis of smoking attributable mortality and costs of infant neonatal care, are described, along with design and data management features and possible applications of the software for policy and program development at various levels of the health care system.
\end{abstract}

(Tobacco Control 2000;9(Suppl III):iii12-iii15)

Keywords: smoking; pregnancy; costs

It is clear that, for the general population in the USA, there is excess morbidity and mortality attributable to smoking. Unfortunately, although considerable progress has been made in measuring the health care costs of smoking that occur in the long run, less is known about the smoking attributable costs that occur when pregnant women smoke. The causal association between maternal smoking and maternal morbidity, infant mortality, and infant morbidity is well established. In $1997,11 \%$ to $23.9 \%$ of pregnant women in the USA reported smoking throughout their pregnancy.

A microcomputer software program (Smoking Attributable Mortality Morbidity and Economic Costs, or SAMMEC) was developed previously by the Centers for Disease Control and Prevention (CDC) to generate national and state estimates of the cost impact of smoking attributable disease. ${ }^{2}$ These impacts are expressed as smoking attributable mortality, years of potential life lost, direct health care costs, and indirect costs. SAMMEC-generated estimates are often used to support tobacco control legislation and policy at the state and national level.

Efforts are currently under way to develop SAMMEC 4.0 for each of three population groups: adults (ages 18 and over), neonates (newborns), and children (ages 0-12 years). The current version of SAMMEC (3.0) does not include estimates of the costs related to smoking during pregnancy. To address this important source of smoking related expenditure, construction of neonatal and childhood components was undertaken as a part of SAMMEC 4.0. In combination, they will form the Maternal and Child Health SAMMEC module (MCHSAMMEC), a stand alone adjunct to SAMMEC 4.0. This user friendly software will allow states and other entities to estimate pregnancy related smoking attributable costs in their population.

\section{Methods}

MCHSAMMEC, as now constructed, performs a prevalence based analysis of smoking attributable mortality and costs of infant neonatal care. Calculation of each of the impact measures relies on the estimate of a smoking attributable fraction (SAF) derived from either a relative risk approach or a multivariate analysis. The use of multivariate analysis to derive the SAF for direct health care costs differs from the attributable risk approach used in the original SAMMEC.

\section{SAF DERIVATION}

The SAF, which is the proportion of cases or deaths that can be regarded as causally linked to cigarette smoking, also represents the proportion by which the outcome would be reduced if exposure to cigarette smoking was eliminated. In our estimates of neonatal costs, or SAFs, exposure includes only active maternal smoking throughout pregnancy. Using responses to the Pregnancy Risk Assessment Monitoring System (PRAMS) question on smoking status during the last three months of pregnancy, and self reports of smoking status during pregnancy from the birth certificate, women were classified as smokers if they indicated that they were still smoking during the third trimester of pregnancy or if they used tobacco during pregnancy. As now constructed, the outcomes in MCHSAMMEC include infant mortality, percentage of infants with low birth weight, birth weight in grams, probability of admission to the neonatal intensive care unit (NICU), number of infant hospi- 
tal days, and total neonatal health care costs. Neonatal health care costs are measured as the dollars paid for all health care services provided to a neonate in the hospital setting. Maternal complications associated with smoking during pregnancy also have the potential to increase health care costs. Previous estimates of these costs used the attributable risk approach and have been published elsewhere. ${ }^{3}$ In our multivariate analysis using PRAMS data, however, we found no effect of active smoking on health care resources used by the mother and hence, these costs are not included in MCHSAMMEC. Indirect costs (for example, lost wages) are also not included at this time.

IMPACT MEASURES: INFANT MORTALITY

Traditionally, derivation of an SAF is based on the following formula, which uses relative risk estimates for smoking related conditions and the prevalence of smoking in the population (here, a pregnant population):

$\mathrm{SAF}=\left\{\left[\mathrm{p}_{0}+\mathrm{p}_{1}\left(\mathrm{RR}_{1}\right)\right]-1\right\} /\left[\mathrm{p}_{0}+\mathrm{p}_{1}\left(\mathrm{RR}_{1}\right)\right]$

where: $\mathrm{p}_{0}=$ percentage of pregnant current nonsmokers; $\mathrm{p}_{1}=$ percentage of pregnant current smokers; and $\mathrm{RR}_{1}=$ relative risk of outcome for pregnant current smokers relative to pregnant current non-smokers.

For infant mortality, the SAF is derived from pediatric conditions (for example, sudden infant death syndrome) resulting in infant death (less than 1 year of age). Again, in estimating these deaths, smoking prevalence refers only to active maternal smoking. For the selected infant conditions, smoking attributable infant mortality is calculated as follows:

smoking attributable infant deaths = total infant deaths $\times$ SAF

The relative risks for infant mortality due to smoking during pregnancy used in deriving this SAF are currently being updated by a review of the literature and estimated by metaanalysis where appropriate. ${ }^{4}$ Methodological details regarding the updated relative risks will be documented and appropriately referenced in the discussion portion of the software (see Design Features section).

IMPACT MEASURES: SMOKING ATTRIBUTABLE DIRECT HEALTH CARE COSTS

The attributable risk approach described above also applies SAFs to corresponding health care expenditures to derive smoking attributable health care costs. ${ }^{2}$ This approach, however, does not account for the complex interactions of factors that determine actual costs incurred by individual smokers (for example, smoker's race, age) or isolate the effects of smoking from other factors, such as alcohol abuse. For example, we do not have estimates of how changing smoking behaviour might change other risk behaviours during pregnancy. Furthermore, we do not know how these behaviours affect the outcomes of interest, birth weight, probability of NICU admission, and infant length of stay either individually or in combination. This approach also does not allow for differences in the costs of a given out- come such as low birth weight for infants of smokers and non-smokers. The multivariate approach corrects for some of these problems and is used here to derive smoking attributable neonatal health care costs for MCHSAMMEC. It is also being used to update the overall SAMMEC 4.0 model and software.

Regression models were used to estimate the relation of maternal smoking to probability of NICU admission and to length of stay for the infant. ${ }^{5}$ Based on these regressions, the formula for the SAF is:

$\Sigma_{\mathrm{i}} \mathrm{w}_{\mathrm{i}}\left[\exp \left(\mathrm{x}_{\mathrm{i}}{ }^{\prime} \mathrm{b}_{\mathrm{p}}\right) /\left(1+\exp \left(\mathrm{x}_{\mathrm{i}}{ }^{\prime} \mathrm{b}_{\mathrm{p}}\right)\right)\right.$ $\left.\exp \left(\mathrm{x}_{\mathrm{i}}{ }^{\prime} \mathrm{b}_{\mathrm{e}}\right)\right]-\sum_{\mathrm{i}} \mathrm{w}_{\mathrm{i}}\left[\exp \left(\mathrm{x}_{\mathrm{i}}^{\star \prime} \mathrm{b}_{\mathrm{p}}\right) /\left(1+\exp \left(\mathrm{x}_{\mathrm{i}}^{\star \prime} \mathrm{b}_{\mathrm{p}}\right)\right)\right.$ $\left.\exp \left(\mathrm{x}_{\mathrm{i}}^{*} \mathrm{~b}_{\mathrm{e}}\right)\right] \div \sum_{\mathrm{i}} \mathrm{w}_{\mathrm{i}}\left[\exp \left(\mathrm{x}_{\mathrm{i}}^{\prime} \mathrm{b}_{\mathrm{p}}\right) /\right.$ $\left.\left(1+\exp \left(\mathrm{x}_{\mathrm{i}}{ }^{\prime} \mathrm{b}_{\mathrm{p}}\right)\right) \exp \left(\mathrm{x}_{\mathrm{i}}{ }^{\prime} \mathrm{b}_{\mathrm{e}}\right)\right]$

where $\mathrm{I}$ indexes individuals: $\mathrm{w}_{\mathrm{i}}$ is the observation weight, $x_{i}$ is the vector of independent variable values for individual $I ; b_{p}$ is the estimated vector of coefficients for the probability equation; and $b_{e}$ is the estimated coefficient vector for the log expense equation. The values of the smoking variable(s) have been set to their actual value in $x^{\prime}$ and to zero in $\mathrm{x}_{\mathrm{i}}^{*}$.

The numerator of this expression is the aggregate smoking attributable birth weight in grams, probability of admission to the NICU, and number of infant hospital days (for example, infant length of stay), depending on the coefficients used. For example, it is the difference in expected birth weight given actual in utero smoke exposure and the expected birth weight given no exposure. The actual dollar value of the expense SAF is based on all inpatient health care expenses per night incurred over the infant's full stay. A weighted average of the NICU expenses per night and the non-NICU expenses per night is used. ${ }^{5}$

The regressions underlying the SAFs were estimated with 1995 data from the PRAMS, an ongoing surveillance project of the CDC. In 1995, 13 states (Alabama, Alaska, California, Florida, Georgia, Indiana, Maine, Michigan, New York (excluding New York City), Oklahoma, South Carolina, Washington, and West Virginia) and the District of Columbia participated in PRAMS. The data are generated so as to be representative of all live births in each state although there is state-specific oversampling of certain low income and racial groups. Sample weights are provided with the data. PRAMS data on approximately 25000 live births in the participating states and the District of Columbia were used.

In addition to PRAMS, a secondary data set available to the CDC from the MedStat Corporation is used to estimate the average number of NICU nights incurred by newborns and to attach average health care expenses to NICU and non-NICU nursery nights. The data set is a subset of birth records drawn from the MarketScan $($ database of commercially insured health care claims. It contains data pooled from Medstat's employer/payer customers. The database contains detailed information on services administered and payments made for each service, including 
deductibles and co-payments, for approximately 17000 deliveries from 1995 . The data are not representative of any particular geographic area.

For a full discussion of the regression techniques used to estimate the above coefficients and the economic interpretation of the smoking attributable cost estimates, the user should refer to the appropriate discussion portions of the software as described in the Disease Impact section of this article.

Users of MCHSAMMEC should recognise the limitations of the actual dollar values generated by the software. Since the data and analysis do not include maternal or indirect costs, they underestimate total costs related to active maternal smoking. Further, the default expenditure data are based on private sector payment information, and hence do not represent expenses for other payers such as Medicaid. Users should focus instead on the SAFs generated by the software. Users should apply the SAFs to the current expenditure data for their population in an effort to correct for some of the limitations of the default expenditure data and to generate estimates more appropriate for their population. To enhance further their use, standard errors for the SAFs are presented in the software output.

\section{Description of software \\ DESIGN FEATURES}

SAMMEC 4.0 is a world wide web site version of the earlier SAMMEC PC resident software. The web site (http://www.medicost.com) is accessible from any computer with a functioning internet connection and browser. The site has been tested and is guaranteed to work with Netscape Navigator 4.0, Microsoft Internet Explorer 4.0, and more recent versions of these products; it does not use any special features that are not present in earlier versions of these and other browsers. Given a connection and a browser, the only additional requirement is a

Table 1 Sample neonatal health care cost smoking attributable fraction: MCHSAMMEC module, SAMMEC Version 4.0

\begin{tabular}{|c|c|c|}
\hline \multicolumn{3}{|c|}{$\begin{array}{l}\text { States selected: } \\
\text { Alabama and Missouri and North Carolina and Wyoming }\end{array}$} \\
\hline \multicolumn{3}{|c|}{ Population demographic selected: } \\
\hline Age (years) & \multicolumn{2}{|c|}{$<18$ or $18-34$ or $>34$} \\
\hline Education & \multicolumn{2}{|c|}{$\begin{array}{l}\text { No high school graduate or high school graduate, or some college or } \\
\text { college graduate }\end{array}$} \\
\hline Insurance & \multicolumn{2}{|c|}{ Private or Medicaid or other public or none } \\
\hline Marital status & \multicolumn{2}{|c|}{ Married or unmarried } \\
\hline Prenatal care & \multicolumn{2}{|c|}{ None or 1 st trimester or 2 nd trimester or 3 rd trimester } \\
\hline Race & \multicolumn{2}{|c|}{ White or African American or other } \\
\hline \multicolumn{3}{|c|}{ Descriptive statistics on selected subset of the standard population: } \\
\hline Observations: & 241015 & Number of observations matching selections above \\
\hline Smoking prevalence: & $16.43 \%$ & Smoking prevalence in selected population \\
\hline SA expenditures: & $\$ 28.48 \mathrm{M}$ & $\begin{array}{l}\text { Smoking attributable expenditures in selected } \\
\text { population }\end{array}$ \\
\hline Total expenditures: & $\$ 1.029 \mathrm{~B}$ & Total expenditures in selected populations \\
\hline SAF among smokers: & $15.22 \%$ & $\begin{array}{l}\text { Smoking attributable fraction of expenditures for } \\
\text { smokers in selected population }\end{array}$ \\
\hline Overall SAF: & $2.77 \%$ & $\begin{array}{l}\text { Overall smoking attributable fraction of expenditures } \\
\text { in selected population }\end{array}$ \\
\hline \multicolumn{3}{|c|}{ User adjusted statistics: } \\
\hline $\begin{array}{l}\text { User's smoking } \\
\text { prevalence: }\end{array}$ & $30.0 \%$ & User's smoking \\
\hline \multicolumn{3}{|l|}{ User adjusted overall } \\
\hline SAF: & $5.05 \%$ & $\begin{array}{l}\text { Smoking attributable fraction of expenditures adjusted } \\
\text { for user's prevalence }\end{array}$ \\
\hline
\end{tabular}

Data are for illustration purposes only. M, million; B, billion. valid user identification and password, which will be supplied by CDC.

CALCULATION OF DISEASE IMPACT

The web site offers health impact measures for each of three population groups: adults (ages 18 years and over), neonates (newborns), and children (ages $0-12$ years). Health impact measures for adults are based on their own smoking history; those for neonates are based on exposure of fetuses to maternal smoking during pregnancy; and measures for children are based on exposure to maternal smoking in the home. Smoking attributable costs for adolescents (ages 13-17 years) are excluded because there are no data to support a simultaneous assessment of the impact of their own smoking and that of exposure to parental smoking. The four measures are smoking attributable mortality, years of potential life lost to smoking; indirect mortality costs, and direct health care costs. The first three of these measures were available in SAMMEC 3.0 and were based on an attributable risk formula as noted previously. The fourth measure, direct health care costs, was based on an attributable risk calculation in SAMMEC 2.0 and, as noted, is absent in SAMMEC 3.0. In SAMMEC 4.0, direct health care cost calculations are based on a series of new econometric models.

The model used to derive direct health care costs for neonates in MCHSAMMEC was briefly described above. In addition, more specific information on this model and other methodological issues underlying MCHSAMMEC estimates is included in the discussion portion of the software. A discussion choice is available to the user for each impact measure.

DATA INPUT

Calculation of the neonatal measures in the MCHSAMMEC module allows the user to specify certain input data. For the direct health care costs, input data are limited to geographic and other population subset specifications plus an optional smoking prevalence override. For example, a user may choose to calculate estimates for a population of Medicaid recipients $20-24$ years old in a certain state. Users also may input their own organisation's or state's expenditure data instead of relying on the estimates derived from the MarketScan ( database described above. For example, a Medicaid user of MCHSAMMEC may want to enter Medicaid expenditures.

In the absence of user supplied data, the web site provides default values for all input data. For the attributable risk-based measures, these are the same data supplied as defaults by the SAMMEC 3.0 software.

Users of SAMMEC 4.0 may store the customised input data on their computers. The "save to file" options allows the user to choose the folders and filenames under which their data are saved. The "retrieve" option can be used to recall input data from previously saved files. 
DATA OUTPUT

For each measure, SAMMEC 4.0 produces a menu of report tables and, in some cases, charts that may be accessed on screen and, optionally, printed or saved to files on the user's computer. The attributable mortality reports available in the MCHSAMMEC module include tables detailing smoking prevalence, relative risk, mortality, and SAFs and smoking attributable mortality. The neonatal health care cost reports include the SAF table (table 1) and the smoking attributable expenditures table which provides both total and smoking attributable expenditures.

\section{Discussion}

Although there have been numerous studies of the increased risk of certain clinical outcomes associated with smoking during pregnancy, ${ }^{67}$ only a few studies have tried to estimate some of the costs related to smoking during pregnancy. A recent issue of Morbidity and Mortality Weekly Report provided the first national estimates of pregnancy related costs attributable to smoking. ${ }^{8}$

The MCHSAMMEC estimates may be used in a number of ways to influence policy and program initiatives. Estimates of health care costs due to smoking during pregnancy are informative to employers who self insure, insurance companies, and managed care organisations. The magnitude of these costs is also important for public programs such as Medicaid, which now covers a large proportion of pregnant women and children. State Medicaid directors can use estimates of direct health care costs and of cost savings associated with smoking cessation to encourage increased coverage of smoking cessation counselling for pregnant women. They could also be used to argue for directing tobacco settlement dollars into the promotion and implementation of smoking cessation programs for pregnant women. Managed care entities may use estimates of cost savings as they consider initiating or expanding programs in prenatal smoking cessation. It is also possible to use MCHSAMMEC estimates to explore the relative cost effectiveness of various interventions and services offered during the prenatal period.

Users of the MCHSAMMEC estimates should recognise the effect of several limitations. As noted, maternal costs, indirect costs, and infant costs beyond the neonatal period are not yet included. Without these costs, MCHSAMMEC underestimates the true costs associated with smoking during pregnancy. Efforts are currently under way to estimate infant costs beyond the costs of delivery as well as those occurring for children exposed to smoke.

The relation between resource utilisation and smoking behaviour, and hence the SAFs, is based on 1995 data from PRAMS. Although PRAMS includes questions on demographics, health status, smoking history, obstetric history, and pregnancy outcomes, it does not include information on pregnancy and childbirth costs. However, there are questions about the length of stay, and whether the infant was admitted to an NICU. In the absence of cost data, these variables can serve as reasonable proxies for resource utilisation and cost.

Because MCHSAMMEC derives expenditure estimates based on the 1996 Market Scan (C) database, users must supply their own expenditure data to obtain user specific dollar estimates. Otherwise, given variations in insurance service coverage and payment rates as well as inflation over time, the dollar estimates derived from the software will not reflect actual costs for that user.

\section{Summary}

SAMMEC 4.0 and its MCHSAMMEC module provide, for the first time, an opportunity for state and other policy and program directors to generate estimates of costs attributable to smoking during pregnancy. The software allows specification of various populations, subsets of populations, smoking prevalence, and expenditure data while maintaining default values for users who may not have this information for their state or organisation. Furthermore, MCHSAMMEC allows users to compare their costs with costs in other states or relevant populations. The utility of MCHSAMMEC will be further enhanced when infant costs beyond the costs of delivery and maternal costs are included.

1 Colley Gilbert BJ, Johnson CH, Morrow B, Gaffield ME, Ahluwalia I, and PRAMS Working Group. Prevalence of selected maternal and infant characteristics, pregnancy risk assessment system (PRAMS), 1997. CDC Surveillance Summaries, September 24, 1999. MMWR Morbid Mortal Wkly Rep 1999;48(No. SS-5):1-37.

2 Shultz JM, Novotny TE, Rice DP. Quantifying the disease impact of cigarette smoking with SAMMEC II software. Public Health Reports 1991;106:326-33.

3 Adams EK, Melvin C. Health care costs attributable to maternal conditions affected by smoking during pregnancy: a combination of meta analysis and claims data. Am F Prev Med 1998;15:212-18.

4 Gavin NI, Wiesen C, Layton C. Review and meta-analysis of the evidence on the impact of smoking on perinatal conditions built into SAMMEC II. Report to the Center for Disease Health, RTI Project No. 7171-010, February 2000.

5 Adams EK, Miller V. Development of the maternal and child health $(\mathrm{MCH})$ smoking attributable morbidity, mortality and economic cost (SAMMEC) model. Final report to the Robert Wood Johnson Foundation (under grant 022247), May 1999

6 Castles A, Adams EK, Melvin CL, Kelsch C, Boulton ML. Effects of smoking during pregnancy. Five meta-analyses. Am f Prev Med 1999;16:2089-15.

7 DiFranza JR, Lew RA. Effect of maternal cigarette smoking on pregnancy complication and sudden infant death syndrome. F Fam Pract 1995;40:1-10

8 Centers for Disease Control and Prevention. Medical care expenditures attributable to cigarette smoking during pregnancy-United States, 1995. MMWR Morbid Mortal Wkly Rep 1997;46:1048-50. 